\title{
Isotopic distribution and dependency to fission product kinetic energy for ${ }^{241} \mathrm{Pu}$ thermal neutron-induced fission
}

\author{
S. Julien-Laferrière ${ }^{1,2, \star}, G$. Kessedjian ${ }^{2, \star \star}, O$. Serot $^{1, \star \star \star}, A$. Chebboubi $^{1}, D$. Bernard ${ }^{1}, A$. Blanc $^{3}$, \\ U. Köster ${ }^{3}$, O. Litaize ${ }^{1}$, T. Materna ${ }^{4}$, O. Meplan ${ }^{2}$, M. Rapala ${ }^{4}$, and C. Sage ${ }^{2}$ \\ ${ }^{1} C E A, D E N, D E R, S P R C, F-13108$ Saint-Paul-Lez-Durance, France \\ ${ }^{2}$ LPSC, Université Grenoble-Alpes, CNRS/IN2P3, F-38026 Grenoble, France \\ ${ }^{3}$ ILL, F-38042 Grenoble, France \\ ${ }^{4}$ CEA, DSM, IRFU, SPhN, F-91191 Gif-sur-Yvette, France
}

\begin{abstract}
Nuclear fission yields data measurements for thermal neutron induced fission of ${ }^{241} \mathrm{Pu}$ have been carried out at the ILL in Grenoble, using the Lohengrin mass spectrometer. The relative isotopic yields for the masses 137 up to 141 have been derived with the associated experimental covariance matrices. Moreover, from preliminary results for the masses 92, 138 and 139, a clear evolution over fission product kinetic energy of the isotopic total count rate is observed.
\end{abstract}

\section{Introduction}

The isotopic fission product yield, $Y(A, Z)$, is the production rate by fission of a specific nucleus of mass $A$ and nuclear charge $Z$. The fission product yields knowledge for thermal neutron induced fission, condensed in the recent evaluated nuclear data libraries (JEFF-3.1.1, ENDF/B-VII.1, JENDL$4.0 \ldots$ ), is one of the major contributor to the uncertainty on computed reactor physics quantities (see for example [1]). Furthermore, the uncertainty propagation can not be properly done since the yields variance-covariance matrices are missing from the evaluated data libraries, despite recent efforts to tackle this issue [2][3][4][5]. Additionally, some discrepancies between the major libraries exist and need to be understood. For these reasons, a collaboration of the LPSC, the CEA and the ILL focuses on producing precise measurement of fission yields for major actinides with the related experimental variance-covariances matrices [6]. In this document, focus will be given to the ${ }^{241} \mathrm{Pu}$ thermal neutron induced fission isotopic yields. The experimental setup will be presented in Sect. 2, followed by the analysis procedure in Sect. 3. The main results will then be displayed and discussed in the last section, Sect. 4.

\section{Experimental setup}

The Lohengrin recoil mass spectrometer [7][8] of which a descriptive figure is presented Fig. 1, has been built in the 70's at the Institut Laue-Langevin (ILL) and is since a major instrument for nuclear

\footnotetext{
${ }^{\star}$ e-mail: sylvain.julien-laferriere@cea.fr

$\star \star$ e-mail: kessedjian@1psc.in2p3.fr

$\star \star \star$ e-mail: olivier.serot@cea.fr
} 


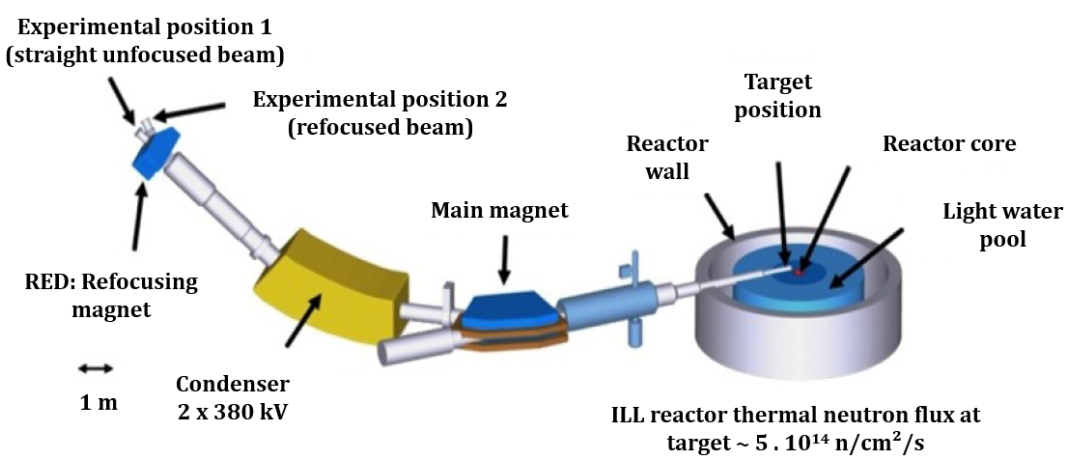

Figure 1. Lohengrin mass spectrometer at the ILL, Grenoble. The ILL high neutron flux compensates the small geometrical acceptance of the instrument.

data measurements in particular due to its high mass resolution $(\Delta A / A \sim 1 / 400)$. A fission target is placed close to the reactor core under a high thermal neutron flux $\left(\sim 5 \cdot 10^{14} \mathrm{n} / \mathrm{s} / \mathrm{cm}^{2}\right.$ at target position). The target undergoes a significant amount of fissions and part of the fission products enters the apparatus (solid angle $\leq 3.2 \cdot 10^{-5} \mathrm{sr}$ ). Through a magnetic and an electric field an ion beam is selected according to respectively the $A / q$ and $E_{k} / q$ ratios, where $A$ is the fission product mass, $E_{k}$ its kinetic energy and $q$ its ionic charge. The ratios $A / q$ and $E_{k} / q$ can be achieved with different triplet $\left(A, E_{k}, q\right)$. To solve this degeneracy, two experimental positions exist: for the position 1 , the beam directly enters an ionization chamber. This position is used to measure isobaric yields, $Y(A)$. For the position 2, the beam is refocused by the Reverse Energy Dispersion (RED) magnet [7], increasing particle density by 7 at maximum, and deposited on a tape. Two clovers of 4 high purity germanium detectors each, are placed in the vicinity of the tape and detect $\gamma$-rays resulting from the fission products $\beta^{-}$disintegration. Knowing the $\gamma$-energies for each wanted fission product $(A, Z)$, one can identify the signature of the decay of a specific nucleus, removing the previous degeneracy. Since the total length of the main path in Lohengrin is $23 \mathrm{~m}$, the fission product time-of-flight is $\sim 1-2 \mu \mathrm{s}$. With additional limitation due to measurement time, only fission products with half-life from a few $\mu$ s to a few hours can be detected by this method. Further constrains, such as the ILL background, also limit the amount of visible nuclides at Lohengrin.

\section{Analysis procedure}

\subsection{Count rate extraction}

Since Lohengrin selects $\left(A, E_{k}, q\right)$ triplets, in order to measure a yield for a specific mass, one has to know both the ionic charge and kinetic energy distributions of this mass. At first, the ionization chamber in experimental position 1, see Fig.1, is used to measure the kinetic energy distribution of the wanted mass. Thanks to Lohengrin, this mass is then selected at the maximum of its kinetic energy distribution. A complete scan of the ionic charge distribution is made at experimental position 2, see Fig.1. For every charge, the integral of the selected $\gamma$ peaks is evaluated, $N_{\gamma}\left(q, A, Z \mid E_{k}\right)$ (see Fig. 2), and corrected from the detector efficiency at the peak energy, $\epsilon_{\gamma}$, and the $\gamma$-intensity, $I_{\gamma}$. The consistency of this corrected count distributions, $N_{\gamma}^{\text {corr }}\left(q, A, Z \mid E_{k}\right)$ (Fig. 3), between the $n \gamma$-rays of the same nucleus is checked through a $\chi^{2}$ test and the mean value is computed (Eq. 1, where $C$ is the covariance matrix [9]). Between each measurement, a background measurement is also performed to be sure to take any residual decay coming from isotopes deposited on the vicinity of the apparatus into account, (particularly important for long-lived nuclides). This happens because the RED magnet does not perfectly refocus the ion beam. The time evolution of the fissile material quantity during 


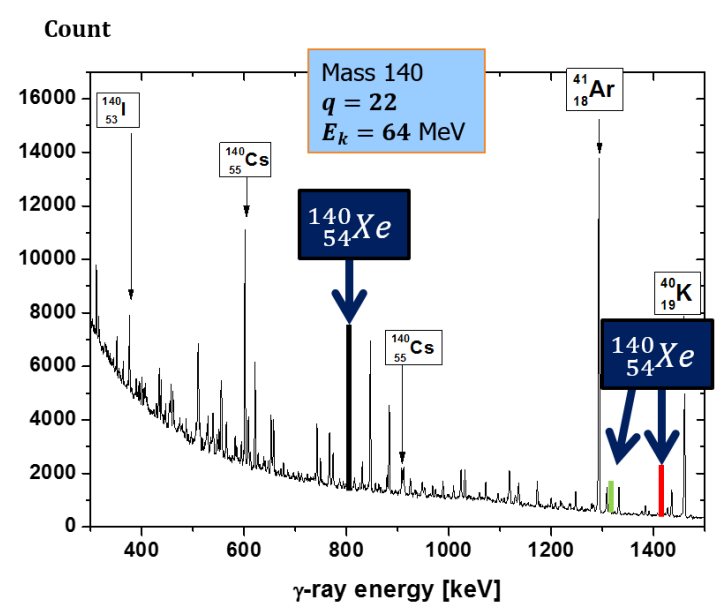

Figure 2. Typical $\gamma$-spectra obtained for the mass 140 at ionic charge 22 and kinetic energy $64 \mathrm{MeV}$. The identification of each nuclei (here in particular ${ }^{140} \mathrm{Xe}$ ) is unambiguous.

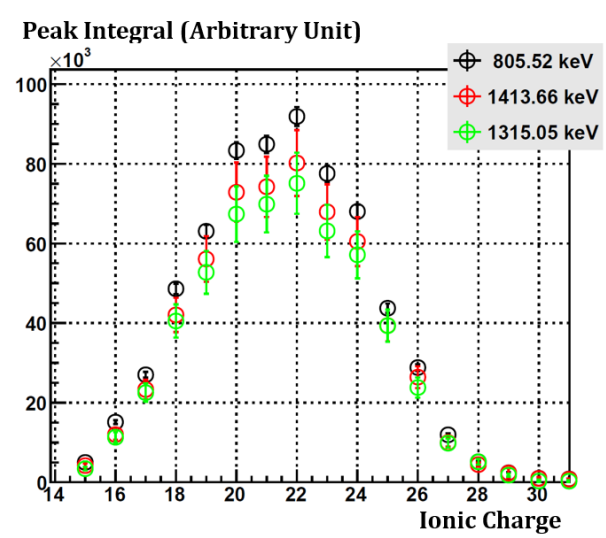

Figure 3. Ionic charge distributions of the integral of each selected $\gamma$-peak for ${ }^{140} \mathrm{Xe}$, corrected by the detector efficiency and the $\gamma$ intensity. Within the uncertainties the distributions are consistent with each other.

the experiment, the Burn-Up (BU), is also carefully estimated by the overall ionic charge and kinetic energy measurements of mass 136 , in order to normalize each measurement.

$$
\begin{aligned}
& N_{\gamma}^{\text {corr }}\left(q, A, Z \mid E_{k}\right)=\frac{N_{\gamma}\left(q, A, Z \mid E_{k}\right)}{\epsilon_{\gamma} \cdot I_{\gamma} \cdot B U(t)} \\
& \overline{N^{c o r r}}\left(q, A, Z \mid E_{k}\right)=\left(\sum_{i, j}^{n, n}\left(C^{-1}\right)_{i, j}\right)^{-1}\left(\sum_{i, j}^{n, n}\left(C^{-1}\right)_{i, j} \cdot N_{\gamma_{j}}^{c o r r}\left(q, A, Z \mid E_{k}\right)\right)
\end{aligned}
$$

\subsection{Relative isotopic yield computation}

Since the independent yields are aimed to be measured, one has to take into account the decay chain between nuclei of the same mass and thus solve the Bateman equations to deduce each independent production rate, $P\left(q, A, Z \mid E_{k}\right)$. The production rate is summed over the ionic charge distribution and the relative isotopic yield is then defined by normalizing the production rate of one mass to 1 , see Eq. 2.

$$
Y\left(A, Z_{i} \mid E_{k}\right)=\frac{\sum_{j} P\left(q_{j}, A, Z_{i} \mid E_{k}\right)}{\sum_{j, l} P\left(q_{j}, A, Z_{l} \mid E_{k}\right)}
$$

\section{Results and discussion}

\subsection{Isotopic yields}

To illustrate this work, the isotopic relative yield distribution for mass 140 is shown Fig. 4 and compared to JEFF-3.1.1. The uncertainties are mainly due to the $\gamma$-rays intensity uncertainties. The results 


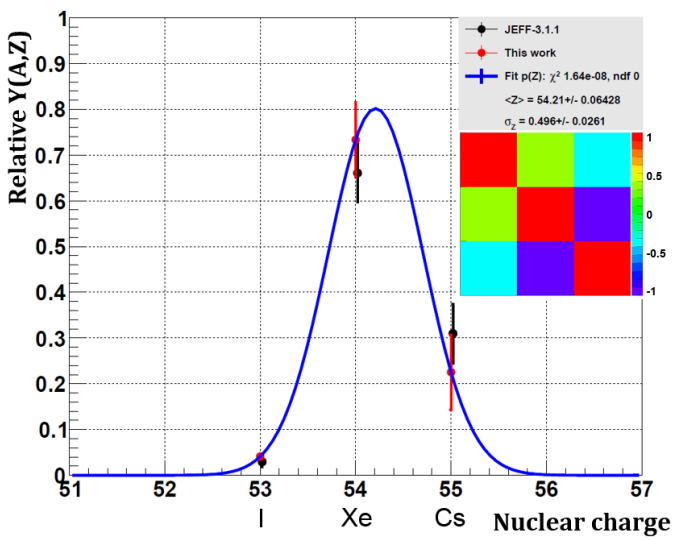

Figure 4. Relative isotopic yield $Y(A, Z)$ for the mass 140 obtained for this work (in red) compared to JEFF3.1.1 (in black) and the related covariance matrix. A Gaussian fit (in blue) is made to extract the mean and the width of the distribution, $<Z>$ and $\sigma_{Z}$.

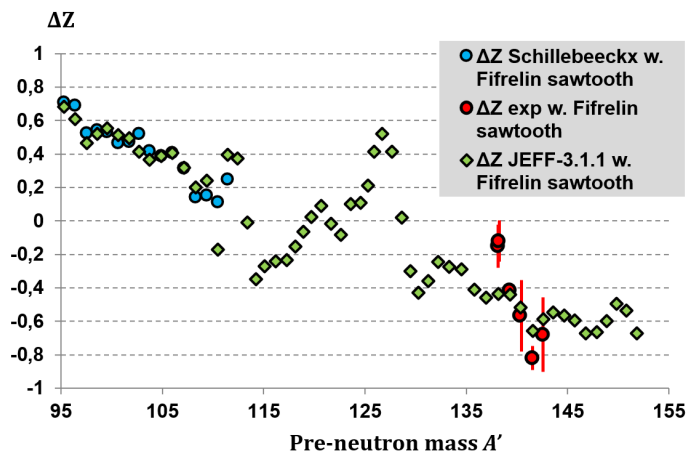

Figure 5. Charge polarization obtained using the Fifrelin sawtooth for this work (in red), compared to JEFF3.1.1 (in green) and Schillebeeckx [11] (in blue) as a function of the fragment pre-neutron emission mass.

Table 1. Relative isotopic yields of the detected nuclei for masses 137 to 141 with their uncertainty. Within a mass the isotopic yields are normalized to 1 .

\begin{tabular}{c|ccc}
$\mathrm{A}$ & $Z$ & $Y(A, Z)$ & $\sigma_{Y(A, Z)}$ \\
\hline \multirow{3}{*}{137} & 52 & 0.037 & 0.007 \\
\cline { 2 - 4 } & 53 & 0.46 & 0.10 \\
\cline { 2 - 4 } & 54 & 0.50 & 0.11 \\
\hline \multirow{4}{*}{138} & 53 & 0.041 & 0.002 \\
\cline { 2 - 4 } & 54 & 0.73 & 0.08 \\
\cline { 2 - 4 } & 55 & 0.23 & 0.08
\end{tabular}

\begin{tabular}{c|ccc}
$\mathrm{A}$ & $Z$ & $Y(A, Z)$ & $\sigma_{Y(A, Z)}$ \\
\hline \multirow{4}{*}{139} & 53 & 0.17 & 0.02 \\
\cline { 2 - 4 } & 54 & 0.66 & 0.11 \\
\cline { 2 - 4 } & 55 & 0.15 & 0.19 \\
\cline { 2 - 4 } & 56 & 0.01 & 0.09
\end{tabular}

\begin{tabular}{c|ccc}
$\mathrm{A}$ & $Z$ & $Y(A, Z)$ & $\sigma_{Y(A, Z)}$ \\
\hline \multirow{3}{*}{140} & 53 & 0.041 & 0.002 \\
\cline { 2 - 4 } & 54 & 0.73 & 0.08 \\
\cline { 2 - 4 } & 55 & 0.23 & 0.08 \\
\hline \multirow{3}{*}{141} & 54 & 0.25 & 0.05 \\
\cline { 2 - 4 } & 55 & 0.69 & 0.09 \\
\cline { 2 - 4 } & 56 & 0.059 & 0.072
\end{tabular}

for the relative isotopic yields for masses 137 to 141 are summarized in Tab. 1, normalized to 1 for each mass independently. Each isotopic distribution can be fitted by a Gaussian to extract for a given mass the mean value, $\bar{Z}$, and the width of the distribution, $\sigma_{Z}$, see Fig.4. Thanks to the sawtooth produced by the Monte Carlo deexcitation code Fifrelin developed by CEA-Cadarache [10], a preneutron mass $A^{\prime}$ can be estimated for each post-neutron mass $A$ and the charge polarization defined as: $\Delta Z\left(A^{\prime}\right)=\overline{Z\left(A^{\prime}\right)}-Z_{U C D}\left(A^{\prime}\right)$ can be computed (knowing that $\overline{Z(A)}=\overline{Z\left(A^{\prime}\right)}$ ). These results are presented Fig. 5 and compared to the JEFF-3.1.1 evaluation and data from Schillebeeckx et al. [11]. A structure is clearly visible in our results whereas JEFF-3.1.1, and results from Schillebeeckx et al. for the light masses present a smoother behaviour. Measurements on a wider mass range could help clarify if it is a local or more general behaviour. The Gaussian fit is also a marker of the completeness of the distribution. In Fig. 4, for $Z=52$ and $Z=56$ a probability close to null is expected from the extrapolation of our data under the hypothesis of a Gaussian distribution. It can reasonably be assessed that the probability of potential missing $Z$ is negligible compared to the measured ones. 

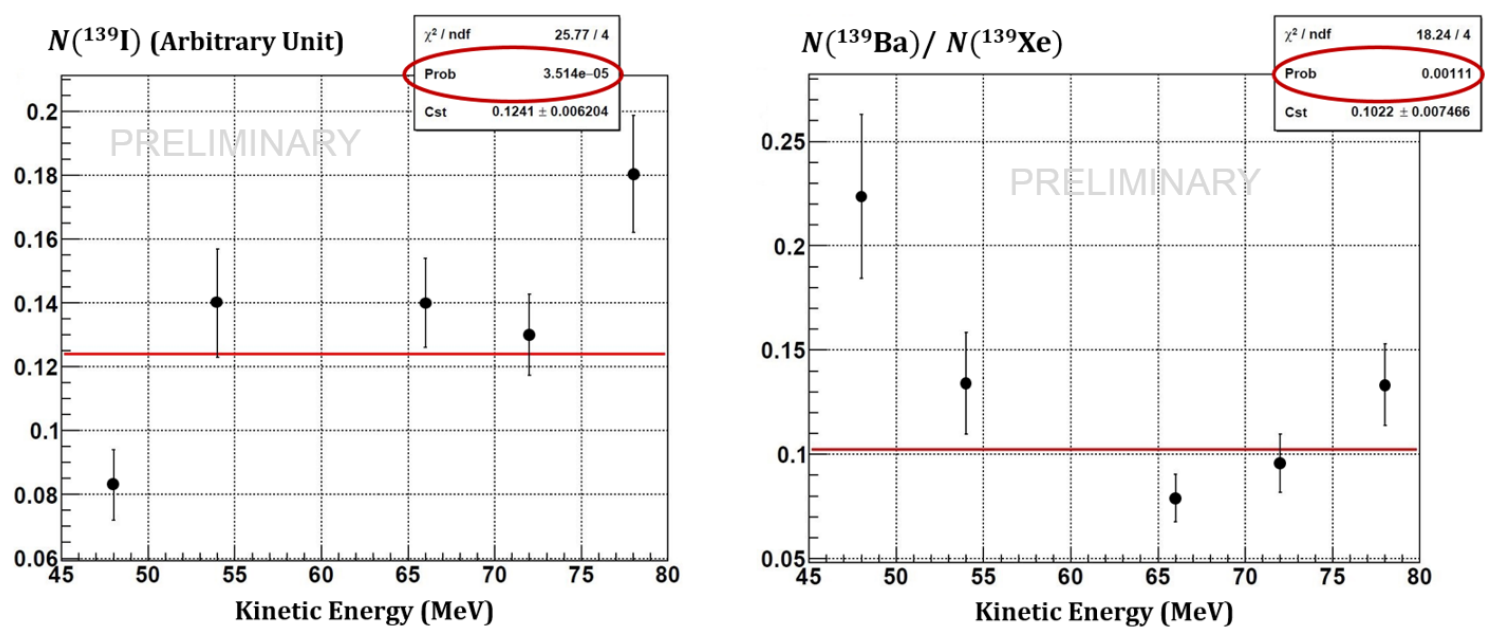

Figure 6. Kinetic energy evolution of the relative total corrected count rate of ${ }^{139} \mathrm{I}$ (on the left) and of the relative total corrected count rate of ${ }^{139} \mathrm{Ba}$ over ${ }^{139} \mathrm{Xe}$ for mass 139 . The constant hypothesis (fit in red) is rejected.

\subsection{Kinetic energy dependency}

Measurements for masses 92, 138 and 139 at different kinetic energies have been made. For low kinetic energy yields, the signal over background ratio is low, therefore, the Bateman equations have not been solved in order to keep reasonable uncertainties and be able to better identify a kinetic energy dependency. Thus, the displayed results are not yields but corrected count rates as defined in Eq. 1 as a function of the fission product kinetic energy. Such count rates are normalized to 1 within a mass for each kinetic energy. Fig. 6 shows these results for ${ }^{139} I$ (on the left) and the ratio of ${ }^{139} \mathrm{Ba}$ over ${ }^{139} \mathrm{Xe}$ (on the right). The associated correlation matrices have also been computed. In view of Fig. 6, a clear correlation between the corrected count rate and the fission product kinetic energy exists. This correlation can be emphasized by the performed constant fit, the small p-values indicate that the constant hypothesis can be rejected at $3 \sigma$ in both cases. The interpretation of these post-neutron emission data is rather difficult since neutron evaporation mixes the initial distribution, no information on the complementary fission product is known and the Bateman equations allowing to get the independent yields have not been solved. Nevertheless, since the constant hypothesis is strongly rejected, a clear effect of the fission product kinetic energy on the relative corrected isotopic count rate is visible. This dependency is currently not included in models used in evaluations and should be investigated and understood. These measurements illustrate the feasibility of a kinetic energy dependency program on Lohengrin in order to test and improve the assumptions of models used in evaluations for all fission observables needed for applications.

\section{Conclusion}

The relative isotopic yields for ${ }^{241} \mathrm{Pu}\left(n_{\text {therm }}, f\right)$ from mass 137 to 141 have been deduced from the ionic charge distribution of each exploitable isotope. All experimental covariance matrices have been determined. More relative independent yields have to be determined along with isomeric ratios for masses 130, 132 and 136. The absolute yields is achievable thanks to mass yield measurements in the IC and is in progress. Finally, with additional work with the Fifrelin code, the observed kinetic energy 
dependency could provide some hints on how the excitation energy at scission is shared between deformation and intrinsic energy.

\section{Acknowledgements}

This work was supported by CEA, IN2P3 and "le défi NEEDS". The authors are grateful for the support of the ILL and all the staff involved from CEA Cadarache and LPSC.

\section{References}

[1] N. Terranova, $\mathrm{PhD}$ thesis, Covariance Evaluation for Nuclear Data of Interest to the Reactivity Loss Estimation of the Jules Horowitz Material Testing Reactor (Università di Bologna, 2016) Chapter 6

[2] N. Terranova, O. Serot, P. Archier, C. de Saint Jean and M. Sumini, Nuclear Data Sheets 123, 225-230 (2015)

[3] N. Terranova, O. Serot, P. Archier, C. de Saint Jean and M. Sumini, accepted for publication in Annals of Nuclear Energy (2017)

[4] L. Fiorito, A. Stankovskiy, G. Van den Eynde, C.J. Diez, O. Cabellos, P.E. Labeau, Annals of Nuclear Energy 88, 12-23 (2016)

[5] D. Rochman, P. Leray, A. Vasiliev, H. Ferroukhi, A.J. Koning, M. Fleming, J.C. Sublet, Annals of Nuclear Energy 95, 125-134 (2016)

[6] O. Serot, C. Amouroux, A. Bidaud, N. Capellan, S. Chabod, A. Ebran, H. Faust, G. Kessedjian, U. Köster, A. Letourneau, O. Litaize, F. Martin, T. Materna, L. Mathieu, S. Panebianco, J.M. Regis, M. Rudigier, C. Sage, and W. Urban, Nuclear Data Sheets 119, 320-323 (2014)

[7] G. Fioni, H.R. Faust, M. Gross, M. Hesse, P. Armbruster, F. Gönnenwein and G. Müzenberg, Nuclear Instruments and Methods in Physics Research A332, 175-180 (1993)

[8] H.R. Faust, P. Geltenbort, F. Gönnenwein and A. Oed, ILL Internal Scientific Report 81FA45S (1981)

[9] M. Schmelling, Physica Scripta 51, 676-679 (1995)

[10] O. Litaize, O. Serot and L. Berge, European Physical Journal A51, 117 (2015)

[11] P. Schillebeeckx, C. Wagemans, P. Geltenbort, F. Gönnenwein, A. Oed, Nuclear Physics A580, 15-32 (1994) 